\title{
Two Thermospray Probe Insert Designs That Allow Clogged Probes To Be Rapidly Replaced
}

\author{
Timothy MacMahon \\ IBM, East Fishkill Facility, Hopewell Junction, New York, USA \\ Christopher W. Baker and John C. Miller \\ Scientific Instruments Services, Inc., Ringoes, New Jersey, USA
}

\begin{abstract}
Thermospray has been widely accepted as a reliable technique for the analytical scientist. Many manufacturers provide thermospray as an option on their mass spectrometers. One difficulty experienced with thermospray is the clogging of the probe inserts. This report describes two new probe insert designs that minimize the problems associated with clogged probe inserts. Both of these new designs minimize the expense and downtime associated with clogged probe inserts. The capillary can be easily and quickly replaced to ensure optimum performance. The new fused-silica probe insert design that is described worked adequately; there was, however, some beam instability caused by rapid oscillation of the fused-silica tubing near the tip. This oscillation also caused the tip to become brittle, necessitating frequent replacement. The stainless steel probe insert described did not exhibit these problems. Last, a comparison is made between the two new designs and the standard Kratos thermospray probe insert. (f Am Soc Mass Spectrom 1993, 4, 182-185)
\end{abstract}

$\mathrm{T}$ Thermospray has proved to be a useful method for introducing liquid matrices into a mass spectrometer. It has been widely accepted, especially in the area of pharmaceutical and environmental applications, and is available as an option on many mass spectrometers. Initially, thermospray was regarded as a troublesome and time-consuming technique; improved instrumentation, however, has greatly increased its utility. One of the major problems still encountered during thermospray is the continual clogging of the probe inserts. For the proper nebulization of the solvent at up to $1 \mathrm{~mL} / \mathrm{min}$ flow rates, the probe tip internal diameter must be approximately $100 \mu \mathrm{m}$. This $100-\mu \mathrm{m}$ orifice requires constant attention and is easily clogged. Over time, the thermospray process will eventually deposit enough nonvolatile material from solvents, salts, and samples to cause the $100-\mu \mathrm{m}$ orifice to become restricted and eventually clog [1-3]. Clogging is accentuated when operating around the area of maximum sensitivity where most of the chromatographic solvent has been heated to vapor (takeoff temperature), especially when more concentrated samples are used.

Thermospray is noted for its wide range in sensitivi-

Address reprint requests to Timothy MacMahon, IBM, East Fishkill Facility, Hopewell Junction, NY 12533. ties and detection limits for various compounds. In the positive ion mode, basic molecules typically have much lower limits of detection and better sensitivities than less basic molecules. Measuring a spectrum of a compound of lower sensitivity, especially in a basic solvent system such as acetonitrile, often requires a more contcentrated sample. To complicate matters, polymers, monomers, glues, and resins typically not only have poor sensitivities, but also have a tendency to oligomerize on heating. If some of these more problematic compounds are injected into the thermospray vaporizer, the probes tend to clog often; once a probe is clogged, it is virtually impossible to remove the obstruction and rejuvenate the probe, owing to the small diameter and long length of the probe insert. There are a few methods for unclogging probes, but none have proved successful with any regularity [1].

Replacement of the probes is expensive because an entirely new insert must be purchased. If these probes have to be routinely replaced, the cost can quickly become prohibitive. To circumvent this problem there have been a few alternative designs. The design favored by most manufacturers involves replaceable probe tips. The replaceable probe tips require the use of a special probe and insert that are not easily adaptable to all thermospray interfaces and, in fact, are not available for the system used throughout these experiments from 
its manufacturer. There are a number of other manufacturers who sell these inserts, but, again, they are not readily compatible with all instruments. The replaceable probe tips work well and have a sapphire insert with a variety of apertures available to the user, ranging from about 40 to $150 \mu \mathrm{m}$. Once these tips are clogged, it is recommended that they be replaced. Should these probes ever develop a clog before the removable sapphire tip, the entire insert would have to be replaced.

Another similar design involves the use of laserdrilled apertures that are easily removed and cleaned once they clog [2]. This design requires special ferrules and aperture disks, and care must be taken on assembly because the aperture disks can be easily ruptured. This design, as with the previous design, does not help if the clog does not occur near the tip.

The last design has a very simple and straightforward probe replacement, but the replacement requires the spot welding of the tip and base thermocouples onto a $1.588-\mathrm{mm}$ (1/16 in.) piece of capillary tubing [3]. This tubing is then inserted through a modified Valco fitting into the vacuum chamber.

This report describes two new probe inserts that were designed at IBM and manufactured by Scientific Instrument Services. These probe inserts minimize downtime and expense related to insert clogging and replacement. The two new designs are also compared with a standard Kratos thermospray probe insert.

\section{Experimental}

All liquid chromatographic/mass spectrometric experiments were performed on a Kratos MS25RFA sector mass spectrometer (Kratos Analytical, Ramsey, NJ) in conjunction with either a Spectra Physics $\$ P 8800$ (Mountain View, CA) or an IBM Instruments 9560 ternary gradient liquid chromatograph. The thermospray interface used was a Vestec design built by Kratos. All solvents were filtered through $0.2-\mu \mathrm{m}$ filters before use and were again filtered prior to their introduction to the thermospray vaporizer. Ammonium acetate (Aldrich, Milwaukee, WI) was the volatile salt for all thermospray experiments and was used as supplied, except for the filtering mentioned previously. The initial probe insert design (Figure 1) was constructed by using a variety of fused-silica capillary tubing (Chrompack, Raritan, NJ) with internal diame-

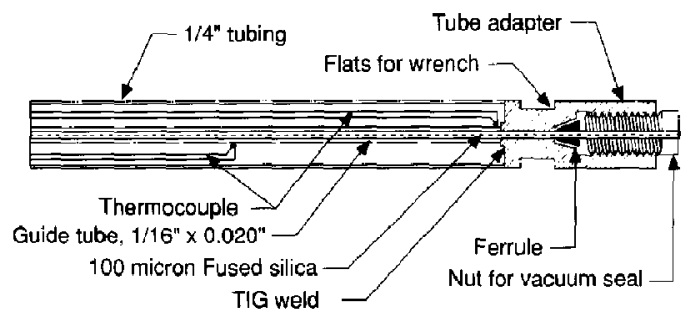

Figure 1. Schematic of the fused-silica probe insert. ters of $25,50,75$, and $100 \mu \mathrm{m}$. Both $75-$ and $100-\mu \mathrm{m}$ fused silica were used throughout these experiments and were used interchangeably. The second probe insert design (Figure 2) used 1.588-mm stainless steel capillary tubing (Upchurch Scientific, Oak Harbor, WA) that had an i.d. of $127 \mu \mathrm{m}$ ( 0.005 in.).

\section{Results and Discussion}

Figure 3 shows the standard Kratos thermospray probe insert with a $1.588-\mathrm{mm}$ stainless steel capillary (i.d. $100 \mu \mathrm{m})$. In this original manufacturer's design, the capillary is silver soldered to the $6.35-\mathrm{mm}(1 / 4 \mathrm{in}$.) stainless steel tubing at the tip, forming the vacuum seal. There are two thermocouples spot welded to the $1.588-\mathrm{mm}$ stainless steel capillary. The first thermocouple is attached to the base and controls the heating, whereas the second thermocouple monitors the temperature at the probe tip. This is the insert type widely used in most thermospray interfaces. This insert is then connected to a $1.27-\mathrm{cm}(1 / 2 \mathrm{in}$.) insertion probe, which is then admitted or withdrawn from the vacuum chamber through a standard $1.27-\mathrm{cm}$ direct insertion lock. Once the capillary tubing is clogged, it is virtually impossible to unclog. Replacement of the capillary tubing requires removal of the silver solder joint, spot welding the thermocouples onto the new capillary, and silver soldering a new vacuum seal at the tip, all of which is very time-consuming and expensive.

Unlike the standard Kratos thermospray probe insert, the two new designs to be described require no spot welding or other modifications when changing probe inserts. Replacement of the capillary tubing in the probe insert is simple and inexpensive.

The fused-silica probe insert (Figure 1) consists of a $6.35-\mathrm{mm}$ stainless steel external tube, a stainless steel guide tube, a stainless steel end fitting, and a $0.21-\mathrm{mm}$ o.d. fused-silica capillary. The end fitting is constructed from a $6.35-1.588-\mathrm{mm}$ tube adapter (Valco Instruments, Houston, TX). The nut from this adapter has a head diameter that is larger than $6.35 \mathrm{~mm}$, making it necessary to modify the nut. The head of the nut was machined to slightly less than $6.35 \mathrm{~mm}$, and flats were then machined into the head so that a wrench could be used during assembly. Flats were also machined into the main hody of this adapter to facilitate assembly.

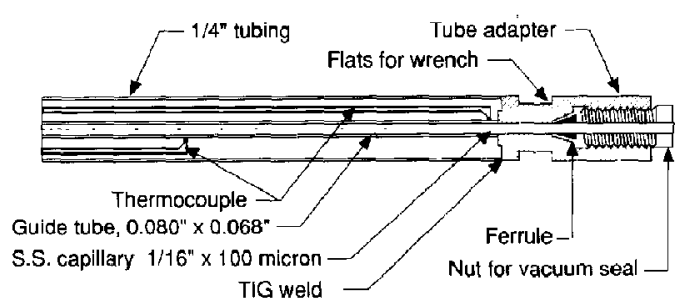

Figure 2. Schematic of the stainless steel probe insert. 


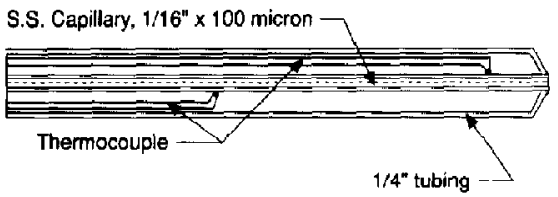

Figure 3. Schematic of the standard Kratos thermospray probe insert.

The stainless steel guide tube is silver soldered into the back of the end fitting. The thermocouples are spot welded to the guide tube in the appropriate locations, and this assembly is tungsten inert gas (TIG) welded onto the end of the $6.35-\mathrm{mm}$ external tube. The guide tube acts as a support for the $0.21-\mathrm{mm}$ o.d. fused-silica capillary tube, which is used to carry the liquid into the mass spectrometer. The fitting and ferrule at the tip of the probe insert form a vacuum seal. The fusedsilica capillary and a low dead volume union are used to connect the fused-silica at the back end of the probe insert to the liquid chromatograph. This entire insert is then connected to the $1.27-\mathrm{cm}$ vacuum probe used with the Kratos insert and again admitted and withdrawn from the vacuum system through the direct insertion lock. When the probe insert clogs, the probe is removed from the vacuum system, and the fusedsilica capillary is replaced by loosening the fitting at the back end and tip.

The stainless steel probe insert (Figure 2) is similar to the fused-silica probe insert (Figure 1), with the exception that a $1.588-\mathrm{mm}$ stainless steel capillary tube is used instead of the fused-silica. The same modified Valco fitting is used at the tip of the probe insert as was described previously. The stainless steel guide tube used for this probe insert design has an i.d. of $1.73 \mathrm{~mm}$ to allow insertion of the $1.588-\mathrm{mm}$ stainless steel capillary. The guide tube is again silver soldered to the end fitting, and the thermocouples are spot welded to the guide tube. The assembly is then TIG welded to the end of the 6.35-mm stainless steel external tube. When the probe insert clogs, the $1.588-\mathrm{mm}$ stainless steel capillary can be quickly replaced by loosening the fittings and sliding another capillary through the guide tube.

When a replacement is needed, a new piece of stainless steel capillary tubing is cut to the proper length by using a standard tube cutter. The only critical part of the procedure is to ensure that the $127-\mu \mathrm{m}$ opening is not closed off while cutting the tube. Kresbach et al. [4] found under similar conditions that the tubing needed to be cut very flat to achieve optimum thermospray conditions; in our experiments, however, no such constraint was observed.

Table 1 shows the various operating parameters for all three probes. In Table 1, the tip temperature and ion counts are separated into two different columns. One column is for straight thermospray and the other for glow discharge-assisted thermospray. All measure-
Table 1. Comparison of the Kratos, fused-silica, and stainless steel probe inserts

\begin{tabular}{lccc}
\hline & Kratos & Fused silica & Stainless ste日l \\
\hline \hline Tip temperature $\left(^{\circ} \mathrm{C}\right)$ & & & \\
Thermospray & 215 & 315 & 235 \\
Glow discharge & 225 & 190 & 200 \\
Base temperature $\left({ }^{\circ} \mathrm{C}\right)$ & 105 & 80 & 129 \\
Source temperature $\left({ }^{\circ} \mathrm{C}\right)$ & 240 & 225 & 220 \\
Jet temperature $\left({ }^{\circ} \mathrm{C}\right)$ & 200 & 200 & 208 \\
LC pump pressure $(\mathrm{bar})$ & 100 & 120 & 105 \\
lon count & & & \\
Thermospray & $10^{2}$ & $10^{2}$ & $10^{2}$ \\
Glow discharge & $10^{4}$ & $10^{4}$ & $10^{4}$ \\
Source pressure (torr) & $10^{-3}$ & $10^{-3}$ & $10^{-3}$ \\
Resolution mass 77 & 474 & 470 & 576 \\
\hline
\end{tabular}

${ }^{a}$ LC, liquid chromatography.

ments for Table 1 were taken with an aqueous $0.1 \mathrm{M}$ ammonium acetate solution pumped into the mass spectrometer at $1 \mathrm{~mL} / \mathrm{min}$. The ion counts were measured by using an ion monitor, located between the electric and magnetic sectors on the mass spectrometer, and give an indication as to the number of ions passing through the electric sector of the instrument.

As can be seen from Table 1, all three probe inserts show similar operating parameters. Both the Kratos probe and the stainless steel probe insert have almost identical operating temperatures, pressures, resolution, and total ion counts. The fused-silica probe insert, however, is significantly different from the other probe inserts in that the temperature necessary for proper thermospray ion production is significantly higher (300 ${ }^{\circ} \mathrm{C}$ versus $220{ }^{\circ} \mathrm{C}$ ).

The difference in the operating temperature of the fused-silica probe insert is probably due to the indirect heat transfer to the liquid matrix. A traditional thermospray vaporizer is heated directly via electrical connections to the inner and outer tubes. The resistivity of the probe insert acts to heat the liquid flowing through it [4]. The fused-silica probe insert cannot be heated directly; fused-silica is an electrical insulator. The electrical connections are made to the outer tube and the guide tube. The liquid in the fused-silica is then heated indirectly via radiative heating through the walls to the silica tubing. This points out the need to properly match the i.d. of the guide tube with the o.d. of the fused-silica. A tight fit between the guide tube and the fused-silica is necessary to maximize the heat transfer to the liquid matrix.

In both the fused-silica and stainless steel probe insert designs, the base and tip thermocouples are not mounted directly on the capillary that is actually carrying the liquid to the mass spectrometer. The thermocouples are attached to the guide tube. It might be expected that they would therefore have a more sluggish response and possibly greater instability [5], but neither of these problems was observed.

The fused-silica probe insert does have a few practical problems. First, because the probe is operated near 
$300{ }^{\circ} \mathrm{C}$, the silica capillary tends to become brittle over time, and the tip can easily break. When the tip breaks it is usually necessary to replace the entire fused-silica capillary. Second, while using this probe insert, it was observed that the vaporizing liquid causes the tip to oscillate at high speeds. This oscillation stresses the tip and makes it further susceptible to cracking. This oscillation also appeared to cause problems with ion beam stability over time. This instability is observed by operating the mass spectrometer in the narrow voltage scan mode. This mode allows the output of the detector to be viewed over a narrow scan range, typically $30,000-100 \mathrm{ppm}$. When the Kratos or stainless steel probe is used, the peak profiles will overlap from scan to scan. When the fused-silica capillary is used, the peak profiles show a difference of about $20 \%$ in peak height from scan to scan.

The exceedingly small i.d. of the guide tube also causes problems when the fused-silica needs to be replaced. Care must be used when removing the old fused-silica and inserting the new fused-silica because the guide tube can become clogged with broken pieces of fused-silica that are difficult to remove.

The stainless steel probe insert design does not suffer from the above-described problems. The capillary is easily replaced, and no clogging of the guide tube is observed. The electrical connections are made directly to the stainless steel capillary, providing excellent thermal properties. The oscillation problems observed with the fused-silica probe insert design do not occur with the stainless steel probe insert because of its rigidity.

After reading the previous description of the problems associated with the fused-silica capillary compared with the stainless steel design, one may wonder why the fused-silica capillary should be considered at all. The main advantage to the fused-silica capillary is that the stainless steel capillary is limited in that the smallest diameter available is about $125-\mu \mathrm{m}$. The fused silica comes in a much wider variety of sizes, ranging from $25 \mu \mathrm{m}$ to over $100 \mu \mathrm{m}$. These smaller sizes allow greater variety in the design of an experiment and allow the experimentalist, not the instrument, to determine the experimental conditions.

Finally, the ion counts and spectra obtained for both types of probes are comparable to the Kratos ther- mospray probe in straight thermospray and glow discharge-assisted thermospray ionization modes.

\section{Conclusions}

Thermospray continues to be a useful analytical technique for interfacing liquid chromatographs and mass spectrometers. One of the problems associated with this technique-clogging of the probe insert-has been minimized with the design of the two new probe inserls described in this report. The performance of the two new probe insert designs is satisfactory and provides easy and reliable performance. Clogged capillary tubes in the probe inserts can be quickly and inexpensively replaced with minimal downtime. The fusedsilica probe insert, although adequate, tended to be a little less stable, probably owing to high-speed oscillations near the tip. The fused-silica probe insert also must be used at higher temperatures to achieve proper thermospray ion production, probably owing to the indirect heating of the liquid matrix. This higher temperature requirement can be minimized by properly matching the i.d. of the guide tube and the o.d. of the fused silica. Both designs provide for quick and easy replacement of clogged capillaries. The replacement of the fused-silica capillary is less convenient, however, than the stainless steel capillary, owing to the narrow internal diameter of the guide tube. These new probe insert designs can easily be adapted to any thermospray interface to provide improved thermospray operation.

\section{References}

1. Hsu, F. F.; Edmonds, C. G. Vestec Thermospray Newsletter $1985,1,4$

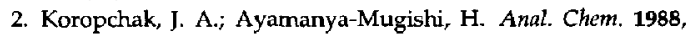
$60,1834$.

3. Unger, S. E.; McCormik, T. J., Bolgar, M. S.; Hunt, J. B. Anal. Chem. 1987, 59, 1242.

4. Kresbach, G. M.; Baker, T. R., Nelson, R. J.; Wronka, J.; Karger, B. L.; Vouros, P. ]. Chromatogr. 1987, 394, 89.

5. (a) Vestal, M. L.; Fergusson, G. J. Anal. Chem. 1985, 57, 2373; (b) Vestal, C. H.; Fergusson, G. J.; Vestal, M. L. Int. J. Mass Spectrom. Ion Proc. 1986, 70, 185; (c) Genuit, W.; Binsbergen, H. V. J. Chromatogr. 1989, 474, 145. 